\title{
An Energy Efficient Model for Monitoring and Detecting Atrial Fibrillation in Wearable Computing
}

\author{
Redjem Bouhenguel, Imad Mahgoub, Mohammad llyas \\ Department of Computer, Electrical Engineering \& Computer Science \\ Florida Atlantic University \\ Boca Raton, Florida, USA \\ rbouheng@fau.edu,mahgoubi@fau.edu,ilyas@fau.edu
}

\begin{abstract}
Current portable healthcare monitoring systems are small, battery-operated electrocardiograph devices that are used to record the heart's rhythm and activity. These on-body healthcare devices fall short on delivering real-time continuous monitoring of early detection of cardiac atrial fibrillation (AFib) when the symptoms last only a short period of time and require a long battery life. The focus of this paper is the design of an energy efficient model for real-time early detection of AFib in a wearable computing device. The design is realized by incorporating an A-Fib risk factor and a real-time A-Fib incidence-based detection algorithm. The results of the design show that the proposed energy efficient model performs better than a telemetry energy model. The design shows promising results in meeting the energy needs of real-time monitoring, detecting and reporting required in wearable computing healthcare applications.
\end{abstract}

\section{Keywords}

Energy-aware model, wearable computing, real-time monitoring, real-time detection of cardiac atrial fibrillation, logistic regression model of atrial fibrillation

\section{INTRODUCTION}

Atrial fibrillation (A-Fib) is the most common cardiac arrhythmia [1] [2] [3]. The American College of Cardiology and the American Heart Association define A-Fib as a supraventricular tachyarrhythmia characterized by uncoordinated atrial activation accompanied by the deterioration of atrial mechanical function. AFib is responsible for approximately 15 percent of the strokes occurring in people with A-Fib. The cost to treat A-Fib in the United States exceeds $\$ 6.4$ billion per year [4]. Small battery operated portable healthcare monitoring systems are used to monitor arrhythmia by recording the heart's rhythm and activities. The recorded data is eventually transmitted either to a physician's office or to a healthcare center for analysis and detection. Unfortunately, these on-body devices are plagued by energy constraints, process optimization problems, data security risks [5] [6] and interference, among others. They need to optimize energy consumption and implement energy management in order to balance innovative interfaces, network resources, continuous monitoring and apropos detection energy requirements.

Copyright 2012, by the Association for Computing Machinery, Inc. Permission to make digital or hard copies of part or all of this work for personal or classroom use is granted without fee provided that copies are not made or distributed for profit or commercial advantage and that copies bear this notice and the full citation on the first page. To copy otherwise, to republish, to post on servers, or to redistribute to lists, requires prior specific permission and/or a fee. Request permission to republish from: Publications Dept., ACM, Inc. Fax +1 (212) 869-0481 or E-mail permissionsacm.org.
The energy consumption must be efficiently allocated. Decisions of which various processes need to run, and when, must be made judiciously in order to deliver essential results in a critical device energy shortage. In addition, wearable healthcare computing devices need the ability to analyze and accurately detect arrhythmia and other medical ailments [7].

This paper presents the design of an incidence-based energy-aware model for real-time detection and reporting of atrial fibrillation in wearable computing devices. Section 1 briefly motivates the need for energy optimization in wearable computing healthcare devices. Section 2 highlights related work. Section 3 describes the required energy components in an A-Fib wearable computing system and defines the incidence, the diagnosis accuracy and the predictors of A-Fib. Section 4 presents the energy models to detect A-Fib, and compares the incidence-based energy-aware model to a telemetry energy model.

\section{RELATED WORK}

Wearable devices face high performance requirements in the middle of energy constraint challenges. Studies and research [8] [9] [10] [11] suggest various methods to minimize power consumption in mobile devices. The authors of [8] describe a framework that is used to reduce the energy consumption of sensors by temporarily turning them off. In study [9], the battery life is extended by as much as $30 \%$ through a collaborative relationship between the operating system and applications. In [10], the authors propose ways to enable systems to trade computational accuracy for resources by scaling down the data or feature set for use on a remote healthcare system. The study reports significant resource savings for small amounts of utility degradation, e.g., $33 \%$ of bandwidth saving for only a $1 \%$ of accuracy degradation. Study [11] suggests a trade-off between power saving and detection accuracy or performance, they show how power can be saved at the loss of a small amount of accuracy by applying different techniques using a low power real-time epilepsy seizure detection algorithm. In project [18], the battery life of a wireless healthcare system is optimized using a dynamic scheduling technique by efficiently assigning tasks to the available resources. The Framingham heart study [17] [20] developed a risk score to calculate an individual's risk of developing atrial fibrillation and a development framework for researcher. The research funded by the Health Technology Assessment Program addresses the accuracy of electrocardiogram (ECG) for the diagnosis of A-Fib and the potential risk of A-Fib misinterpretation errors[12] [13] [14]. Finally, a mobile medical device, dubbed HeartSaver [15] was developed to monitor the onset of atrial fibrillation and other cardiac pathologies. Our design extends battery life in a Risk Incidence-Based energy-aware model that may be applied in wearable computing devices to continuously monitor and detect the onset of A-Fib. 


\section{PRELIMINARIES}

This section describes the required energy components in an A-Fib wearable computing system and defines the incidence, the clinical diagnosis accuracy and the predictors of A-Fib.

\subsection{Devices}

Typically, a wearable computing device requires energy for signal sensing ( $\mathrm{E}_{\mathrm{ECGsense}}$, for Bluetooth signal transmitting from the sensor to the GSM phone $\left(\mathrm{E}_{\mathrm{ECGTx}}\right)$, for GSM phone signal receiving from the Bluetooth sensor $\left(\mathrm{E}_{\mathrm{ECGRx}}\right)$, for GSM phone analysis and detection ( $\mathrm{E}_{\text {classify }}$ ), and for the GSM phone transmitting results $\left(\mathrm{E}_{\mathrm{Rep}}\right)$ (see Figure 1). We compare the energy consumed by a Risk and Incidence Based A-Fib Detection Scheme to the energy consumed by a telemetry model. In this study, we use the two-lead ECG Alive Technologies Heart Monitoring Device A102D7 (650 mAh at $3.7 \mathrm{~V})$ [16]. The device monitors and transmits ECG signals via Bluetooth to an Apple MacBook computer (see Figure 2). We assume that the telemetry model continuously monitors and transmits ECG signals during a 24-hour period.

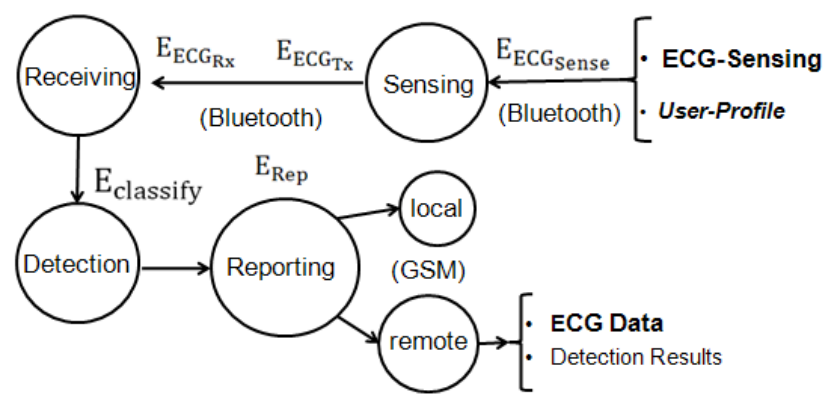

Figure 1: Wearable computing energy functional requirements

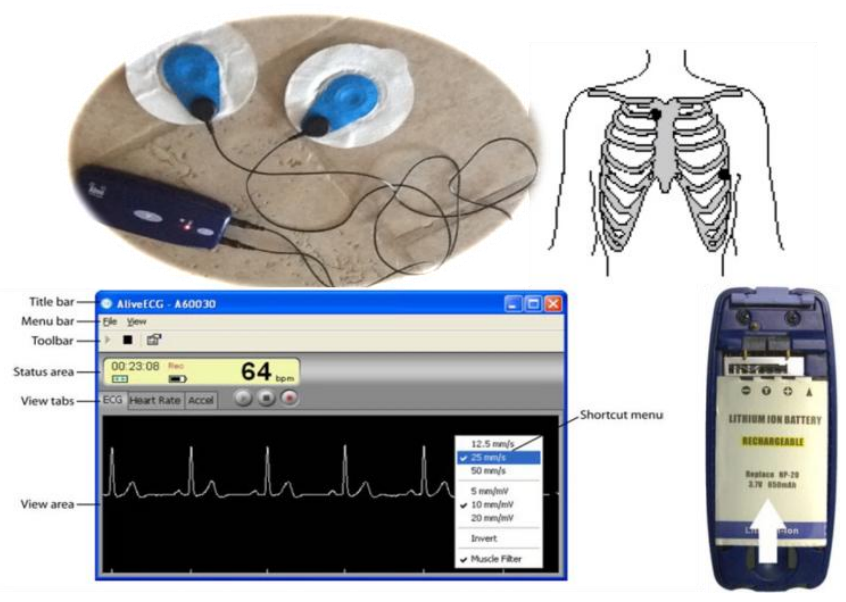

Figure 2: Alive Technologies Heart Monitoring Device A102D7

\subsection{Understanding A-Fib}

The following subsections define the incidence rate of A-Fib, the clinical diagnosis accuracy of the onset of A-Fib, and the predictors A-Fib.

\subsubsection{Incidence Rate of A-Fib}

Among all arrhythmia, A-Fib is the most frequently diagnosed and affects 2.5 million people in the United States, or close to $1 \%$ of the total population [4]. The Manitoba study [37] concluded that the incidence of A-Fib is 0.13 to 0.36 for people between 25 and 60 years old, 5.7 per 1,000 person-years after age 60, and 9.7 per 1,000 person-years after age 70. The Framingham Heart study [36] and other studies draw attention to the significance of the higher frequency of A-Fib with advancing age [19]. Patients with A-Fib have a 1.5-2 fold increase in mortality rate when compared with the general population as suggested by Framingham Heart study data [17] [20]. Early recognition of A-Fib is difficult because most people are not aware of this silent rhythm disturbance [21]. Today, frequent monitoring and screening of patients allow for early detection of arrhythmia.

\subsubsection{Clinical Diagnosis Accuracy of A-Fib}

At least one-third of the A-Fib episodes go undetected because either people do not get screened often, or A-Fib diagnosis is missed by a general practitioner or practice nurse [23]. Few studies have addressed the misdiagnosis of A-Fib from an electrocardiogram (ECG) and the potential risk of A-Fib misinterpretation errors. Knight et al. [13] concluded that A-Fib is more often misdiagnosed by internists than cardiology fellows and cardiologists. Mant et al. [23] discovered that general practitioners correctly detected A-Fib $80 \%$ (true positive) of the time when interpreting 12-lead ECG data and misinterpreted 8\% (false positive) of sinus rhythm cases as A-Fib. One of the major misdiagnosis confuses A-Fib with atrial flutter [13] [24].

\subsubsection{Predictors of A-Fib}

A-Fib is the most prevalent arrhythmia in the United States and accounts for more than 750,000 strokes per year [25]. According to classification guidelines used by cardiologists and electrophysiologists, for the management of patients with A-Fib [26], after the first A-Fib is detected, there are mainly four types of AFib: Paroxysmal, persistent, longstanding persistent, and permanent. A-Fib is termed progressive, as once a patient is diagnosed with a paroxysmal A-Fib, he or she will eventually migrate to persistent A-Fib. Similarly, a patient diagnosed with persistent A-Fib, will drift to longstanding persistent A-Fib and in time to permanent A-Fib [27].

Some of the ECG waves and intervals in figure 3 are used to derive our A-Fib detection algorithm. The latter plays an integral part in the Risk Incidence-Based energy model. The QRS interval is the duration of the ventricular muscle depolarization. The $\mathrm{P}$ wave is a record of the electrical activity or the sequential activation (depolarization) through the right and left atria. The PR interval is the time interval measured from the beginning of the $\mathrm{P}$ wave (atrial depolarization) to the onset of the QRS complex (ventricular depolarization). The RR interval is the duration of the ventricular cardiac cycle; it is an indicator of the ventricular rate. The PP interval is the duration of the atrial cycle; it is an indicator of the atrial rate. 


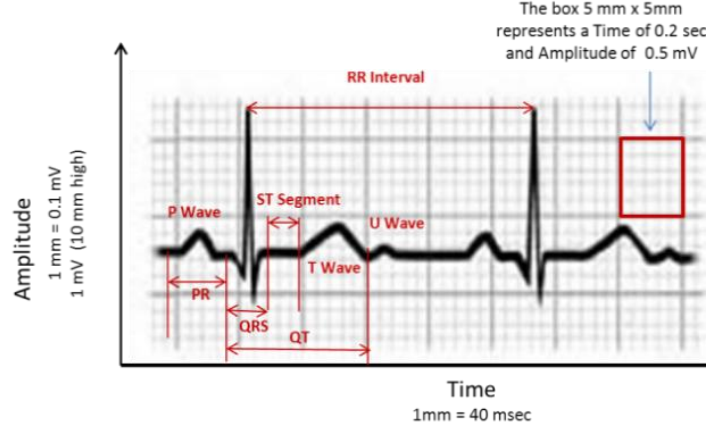

Figure 3: Typical ECG wave and intervals

During A-Fib, the electrical signal begins in a different part of the atria instead of the SA node. The abnormal signal causes the atria to quiver rapidly instead of contracting normally. The atria do not pump blood efficiently into the ventricles causing the blood to pool in the atria where clots can form. Blood clots may travel from the heart to the brain resulting in strokes (Figure 4 describes the path of the electrical signals during an A-Fib episode).

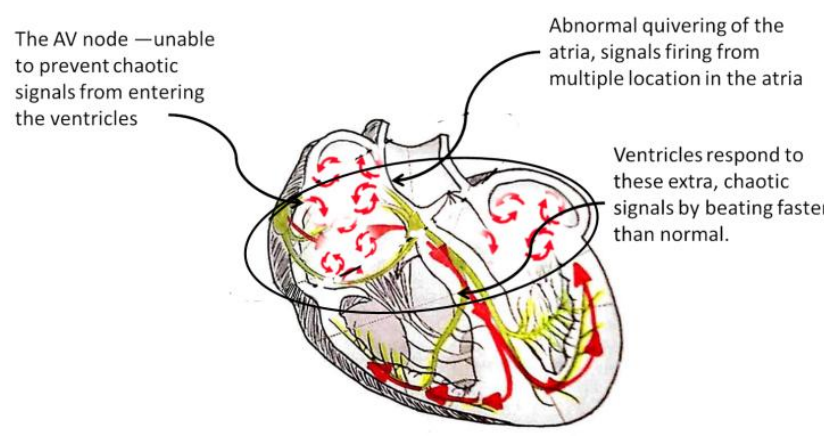

Figure 4: Describing A-Fib chaotic electrical signals

We acknowledge detecting A-Fib is difficult and requires a more intense research, however one of the strong indicators of A-Fib presence is the absence of $\mathrm{P}$ waves on the ECG plot and an erratic noise-like activity in their place combined with irregular R-R intervals [28][25][27]. Sometimes when the heart rate is too fast, irregular RR intervals may be difficult to determine [19]. Wide QRS complexes may be present with rapid ventricular response.

\section{Energy Models to Detect A-Fib}

Today, portable healthcare monitoring devices such as Holter monitors, event monitors, and telemetry devices are small battery operated electrocardiograph (ECG) devices, which are used to monitor a patient's heart activity for periods of time ranging from days to weeks. Sensed or recorded ECG data is sent to a doctor or a care center for analysis and reporting. Unfortunately these on-body devices are not energy efficient; they drain batteries quickly and necessitate patients to replace batteries sometimes daily [29]. This weakness in wearable computing devices runs the risk of missing the first 30 seconds of A-Fib or might not be possible if the user is incapacitated. They also fall short on delivering real-time detection; the patient waits for the eventually transmitted recorded data to be analyzed and results fed back to him. Telemetry and wearable healthcare computing systems are concerned with three main components: monitoring, detection, and reporting. In an energy- aware environment, the different components must run sensibly in order to extend battery life.

\subsection{Telemetry Energy Model}

When prescribed by a physician, telemetry may be applied continuously for few days in the hope of capturing episodes of AFib. Telemetry may also be user-triggered by the patient as soon as he or she feels symptoms of A-Fib (such as heart palpitations). We assume that telemetry ECG interpretations are conducted by a cardiologist or a cardio-physiologist who is a trained expert at ECG readings thus, all judgments of what constitutes A-Fib are going to be assumed to be as accurate as possible. The total energy consumed is the sum of the energies that are required for sensing ECG signals, transmitting to the cell phone via Bluetooth, receiving ECG record, and reporting ECG record for a period of 24 hours.

$\mathrm{E}_{\text {telemetry }}=\mathrm{E}_{\mathrm{ECG}_{\text {Sense }}}+\mathrm{E}_{\mathrm{ECG}_{\mathrm{Tx}}}+\mathrm{E}_{\mathrm{ECG}_{\mathrm{Rx}}}+\mathrm{E}_{\mathrm{Rep}}$

The telemetry report includes all positive and negative results. False positive outcomes are usually interpreted as false alarms; they contribute to wasted or needless energy spent in transmitting inaccurate information. In a 24-hour period, such a telemetry system would use approximately $50 \%$ of the capacity of the Heart Monitoring Device battery. Typical monitoring and detection healthcare wearable body network devices have limited energy and therefore limited monitoring duration.

\subsection{Risk and Incindence Based Energy Model}

The implementation of a risk and incidence based A-Fib detection model in A-Fib monitoring devices alleviates the aforementioned challenges in telemetry[30] [31]. For instance, knowing the A-Fib risk factor of a patient allows one to prescribe an A-Fib monitoring and detection schedule. A high A-Fib risk factor may suggest more frequent monitoring compared to a low A-Fib risk factor. Because A-Fib is not a common occurrence [23], we want to report a result only when there is an actual occurrence of A-Fib. We adopt an AFib logistic regression model to detect the first episode of A-Fib, that is the first 30 seconds of continuous A-Fib. After the first ${ }_{30}$ seconds of A-Fib is detected, monitoring may proceed beyond 24 hours to detect paroxysmal, persistent, long-standing persistent and permanent A-Fib. Monitoring may be prescribed for days or weeks.

\subsubsection{Assessing the A-Fib Risk Factor}

The Cox proportional-hazards regression [32] is used to analyze the effect of risk factors on survival. The probability of the onset of A-Fib is called the hazard. The following covariates and their corresponding coefficients responsible for predicting A-Fib risk in people aged between 45 and 95 years old are extracted from the Framingham Heart Study [17] [20]: Age, $\mathrm{Age}^{2}$, Gender, Body Mass Index (BMI), Systolic Blood Pressure (SBP), Treatment for Hypertension (TH), Significant Heart Murmur (SHM), Prevalent Heart Failure (PHF), Gender*age ${ }^{2}$, and Age*PHF, PR Interval $\left(\mathrm{PR}_{\text {interval }}\right)$. We can express the hazard or risk of getting A-Fib at time $t$ as:

$$
H(t)=H_{0}(t) * e^{\sum_{\mathrm{i}=1}^{\mathrm{k}} \beta_{\mathrm{i}} \mathrm{X}_{\mathrm{i}}}
$$

Where $\mathrm{H}_{0}(10)=0.96337$ is the 10 year baseline survival or cumulative hazard function at time $\mathrm{t}=10$ years extracted from the Framingham Heart study [17] [20]. 


$$
\begin{array}{rl}
\sum_{i=1}^{k} \beta_{i} X_{i}=1.994 & 60 \text { Gender }+0.150520 \text { Age } \\
& +0.019300 \text { BMI }+0.006150 \text { SBP } \\
& +0.424100 \mathrm{TH}+3.795860 \text { SHM } \\
& +9.428330 \text { PHF }-0.000380 \text { Ag }^{2} \\
& -0.000280 \text { Gender } * \text { Age } \\
& -0.042380 \text { Age } * \text { SHM }-0.123070 \text { Age } \\
& * \text { PHF }+0.070650 \text { PR Interval }
\end{array}
$$

For example, the predicted Risk Factor for a male person, who is 70 years old, who weighs $70 \mathrm{~kg}$, with a body mass index of 22.96 , a systolic blood pressure (SBP) of 130, no hypertension, a PR interval measuring of $16 \mathrm{~ms}$, no significant heart murmur, and no previous heart failure, is 0.0863 . This is compared to the risk for a person of the same age and gender, with BMI 20 to 24.9, a normal SBP (120 to 129), no treatment for hypertension, a PR Interval of 16 , no significant murmur or prevalent heart failure.

\subsubsection{Implementing A-Fib Detection}

The dataset used in our analysis was extracted from the Machine Learning Repository at University of California, Irvine [33], MITBIH Atrial Fibrillation database [34] and from data donated and corroborated by a cardiologist. The dataset describes the attributes

\begin{tabular}{|c|c|c|c|}
\hline & Variable & Description & Value \\
\hline 1 & age & Age in years, linear & real \\
\hline 2 & $\mathrm{Age}^{2}$ & $\mathrm{Age}^{2}$ in years ${ }^{2}$ & real \\
\hline 3 & Gender & $\begin{array}{l}\text { Gender }(0=\text { male; } 1=\text { female }) \text {, } \\
\text { nominal }\end{array}$ & $\{0,1\}$ \\
\hline 4 & BMI & $\mathrm{Kg} / \mathrm{m}^{2}$, Linear & real \\
\hline 5 & QRSduration & $\begin{array}{l}\text { Average of QRS duration in } \\
\text { msec., linear }\end{array}$ & real \\
\hline 6 & PRinterval & $\begin{array}{l}\text { Average duration between onset } \\
\text { of } \mathrm{P} \text { and } \mathrm{Q} \text { waves in msec., } \\
\text { linear }\end{array}$ & real \\
\hline 7 & heartrate & $\begin{array}{l}\text { Number of heart beats per min, } \\
\text { linear }\end{array}$ & real \\
\hline & class & $\{$ A-Fib present, A-Fib absent $\}$ & binary \\
\hline
\end{tabular}
for diagnosing cardiac A-Fib where each instance or patient is classified into two categories: presence of cardiac A-Fib and absence of cardiac A-Fib. The resulting dataset contains 304 records including 80 A-Fib cases, 224 non-A-Fib cases, seven attributes and two classes (A-Fib Present, A-Fib Absent). The cardiologist's classification is used as a reference (see Figure 5).

Figure 5: A-Fib attributes.

Logistic regression determines the relative effect of independent variables $\mathrm{x}_{\mathrm{i}}$ on the dependent variable $\mathrm{Y}$ or class and their statistical significance. This effect is usually explained in terms of odds ratios where the odds of an event $x$ occurring with probability $p$ is defined as: odds $(p)=p /(1-p)$ where $p$ is the probability of the presence of the disease. The logit transformation is defined as the natural $\log$ of odds,

$$
\begin{aligned}
& \operatorname{logit}(p)=\ln \left(\frac{p}{1-p}\right) \\
& p(Y=1 \mid x)=\frac{1}{1+e^{-\operatorname{logit}(p)}}
\end{aligned}
$$

$$
\operatorname{logit}(p)=\beta_{0}+\sum_{\mathrm{i}=1}^{\mathrm{k}} \beta_{\mathrm{i}} \mathrm{x}_{\mathrm{i}}
$$

$x_{i}=\left(x_{1}, x_{2}, \ldots, x_{k}\right)$ is the covariate vector and $\beta_{i}(i=1,2, \ldots, k)$ denotes the coefficients of the $\mathrm{k}$ predictors. Fitting a logistic regression model to a given data implies deriving estimates of the coefficients $\beta_{i}$ that maximize the likelihood of the model. The outcomes of the Logistic Regression include all True Positive and False Positive results. They may be triggered at A-Fib incidence rates reported in the Manitoba studies [37] where the incidence of A-Fib is 0.13 to 0.36 for people between 25 and 60 years old, 5.7 per 1,000 person-years after age 60 , and 9.7 per 1,000 person-years after age 70 .

A-Fib is predicted present if probability p (A-Fib is Present $\mid$ age, age $^{2}$, gender, BMI, QRSduration, PRinterval, heartrate) $>0.5$

Otherwise, A-Fib is absent.

$$
\begin{aligned}
\operatorname{logit}(\mathrm{p})= & -41.175+0.820 \text { age }-0.006 \mathrm{age}^{2}+4.737 \text { Gender } \\
& -0.047 \text { BMI }+0.098 \text { QRSduration }-0.178 \text { PRinterval } \\
& +0.066 \text { Heartrate }
\end{aligned}
$$

and $\mathrm{p}=1 /\left(1+\mathrm{e}^{-\log i t}(\mathrm{p})\right)$

Figure 6 describes a possible daily monitoring and detection of episodes of A-Fib according to a logistic regression model.

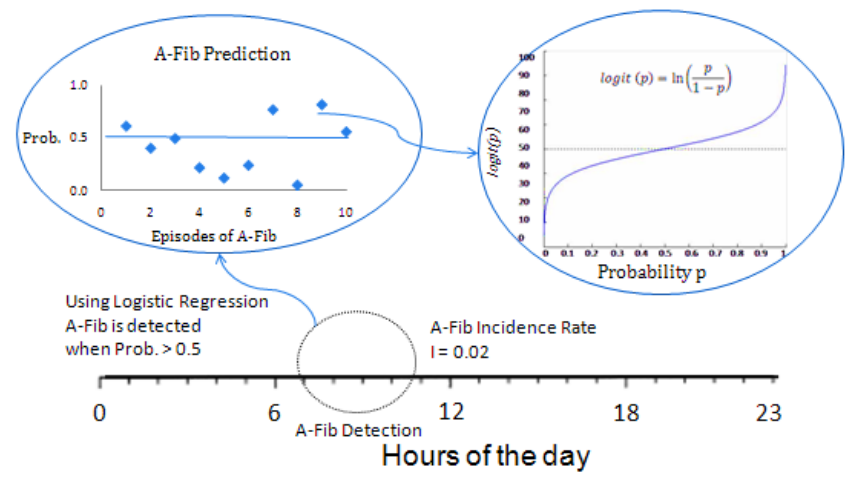

Figure 6: A-Fib Episodes

\subsubsection{Evaluating Classifier Performance}

Given an ECG record, a binary classification has four possible outcomes or rates: True negative (TN), False Positive (FP), True Positive (TP), and False Negative (FN). Detection rates are measured in terms of sensitivity and specificity [26]. Both the overall classification accuracy and the overall classification error defined below may be used to evaluate the performance of the classifier:

$$
\begin{aligned}
& \text { Overall Error rate }=\frac{\mathrm{FP}+\mathrm{FN}}{\mathrm{TP}+\mathrm{TN}+\mathrm{FP}+\mathrm{FN}}=2.63 \% \\
& \text { Overall Accuracy }=\frac{\mathrm{TP}+\mathrm{TN}}{\mathrm{TP}+\mathrm{TN}+\mathrm{FP}+\mathrm{FN}}=97.37 \%
\end{aligned}
$$

However, when the cost of misclassifications of the different classes is uneven, this measure may be unacceptable. In order to take into account the unevenness of misclassification costs when 
evaluating a classifier, area under the Receiver Operating Characteristic (or ROC) curve is explored. ROC curves have been used in biomedical informatics [7] to express the sensitivity versus specificity of classifiers.

The ROC curve plot displays the False Positive rate on the X-axis (1- Specificity) and the True Positive rate (Sensitivity) on the Yaxis. Each point on the ROC curve represents a sensitivity / (1specificity) pair corresponding to a particular decision threshold. The area under the ROC curve measures how well a particular parameter can distinguish between two diagnostic groups (such as presence of a disease/ absence of A-Fib). The bigger the area is and the closest to 1 , the better the classifier performance. The area under the ROC curve for the derived logistic regression model is 0.986 .

The A-Fib detection algorithm is triggered by the onset of A-Fib. Suggested studies [24] reveal that clinical measurement of sensitivity (True Positive rate) of $80 \%$ and specificity (True Negative rate) of $92 \%$ when internists and general practitioners instead of cardiologists diagnose A-Fib. Our logistic regression classification of A-Fib has a measurement of sensitivity of $98.8 \%$ and specificity of $96.9 \%$. The false positive results, usually interpreted as false alarms, contribute to wasted or needless energy spent in transmitting inaccurate information.

\subsection{Applying Risk and Incidence Based Energy Model to A-Fib Detection}

A-Fib monitoring devices may become impractical when they run out of battery energy. Typical monitoring and detection healthcare wearable body network devices have limited energy and therefore limited monitoring duration. The implementation of a risk and incidence based A-Fib detection in such devices helps extends a monitoring device battery life. For instance, A-Fib risk factors may be classified in three categories made up of risk ranges such as $\mathrm{k}<$ $0.05,0.05<\mathrm{k}<0.15, \mathrm{k}>0.15$. Knowing the A-Fib risk factor of a patient allows one to prescribe an A-Fib monitoring and detection schedule (see Figures 7). A high A-Fib risk factor may suggest more frequent monitoring compared to a low A-Fib risk factor.

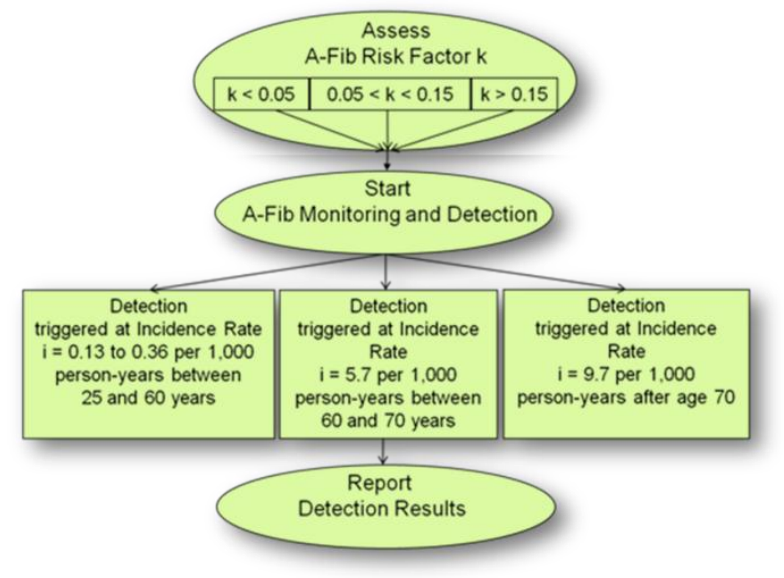

Figure 7: Overview of an efficient wearable computing device

We design an A-Fib detection energy model by adopting an A-Fib risk factor assessment algorithm from [20] and a logistic regression model. We consider an incidence rate equal to $2 \%$ for illustration purposes. The total energy consumed during a 24-hour period is the sum of the following energies, $\mathrm{E}_{\mathrm{ECGsense}}$ for continuously sensing ECG signals, $\mathrm{E}_{\mathrm{ECGTx}}$ for transmitting ECG signals to the cell phone via Bluetooth, $\mathrm{E}_{\mathrm{ECGRx}}$ for receiving ECG signals, $\mathrm{E}_{\text {classify }}$ for classifying the received data, and $\mathrm{E}_{\mathrm{Rep}}$ for reporting when there is an episode of A-Fib suggested by the detection algorithm output at the positive rate $r_{p}$.

$$
\begin{aligned}
\mathrm{E}_{\mathrm{RPclassification}}= & \mathrm{E}_{\mathrm{ECG}_{\text {sense }}}+\mathrm{E}_{\mathrm{ECG}_{\mathrm{Tx}}}+\mathrm{E}_{\mathrm{ECG}_{\mathrm{Rx}}}+\mathrm{E}_{\text {classify }}+ \\
& \mathrm{r}_{\mathrm{p}} * \mathrm{E}_{\mathrm{Rep}}
\end{aligned}
$$

Figures 1 and 8 illustrate the major components in the A-Fib detection energy model.

Ideally, when there are no A-Fib episodes, $r_{p}$ is equal to 0 that is, the model spends its time in a monitoring state. On the other hand, when the model continuously monitors, and continuously transmits, $r_{p}$ is equal 1. False positive outcomes result in wasted energy that is needlessly spent transmitting inaccurate information. In a 24-hour period, such a detection system would necessitate $31.1 \%$ of the capacity of the Heart Monitoring Device battery. This is equivalent to $61.8 \%$ of the energy consumed by a telemetry energy model.

The authors plan to implement a risk and incidence based atrial fibrillation detection scheme in a wearable device and further validate the results in a clinical setting.

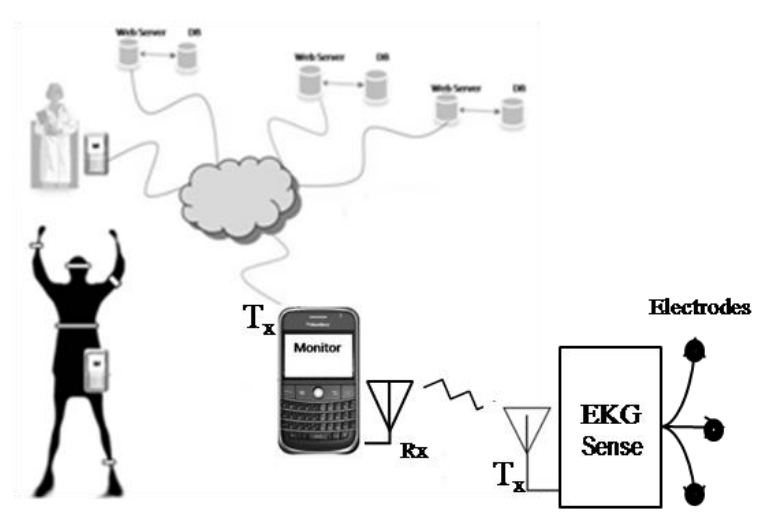

Figure 8: Overview of a wearable A-Fib detection system

Figure 9 illustrates the energy consumption as the positive detection rate varies with respect to the clinical incidence rate.

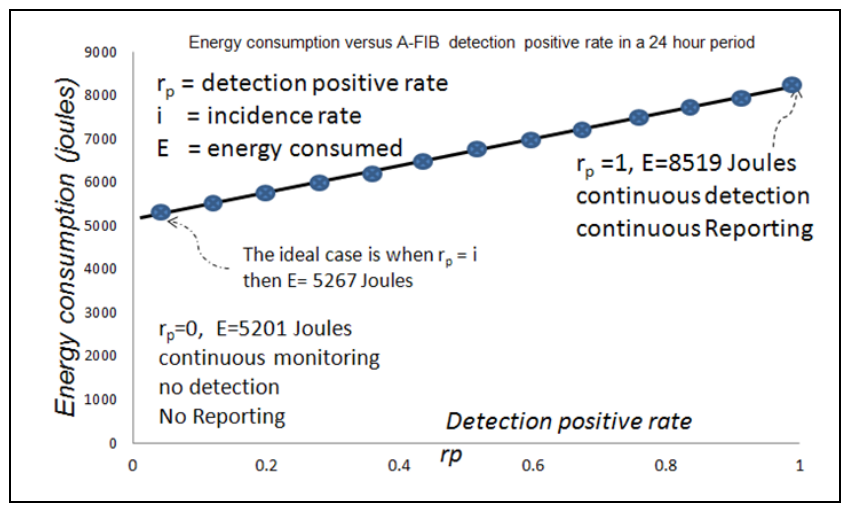

Figure 9: Energy consumption versus A-Fib positive rate $r_{p}$ 
Figure 10 suggests that the ideal detection case is when the logistic regression positive rate $r_{p}$ is equal to cardiologist referenced A-Fib incidence rate $\mathrm{i}$. The worst case is when the positive rate equals 1 , which corresponds to a telemetry energy model.

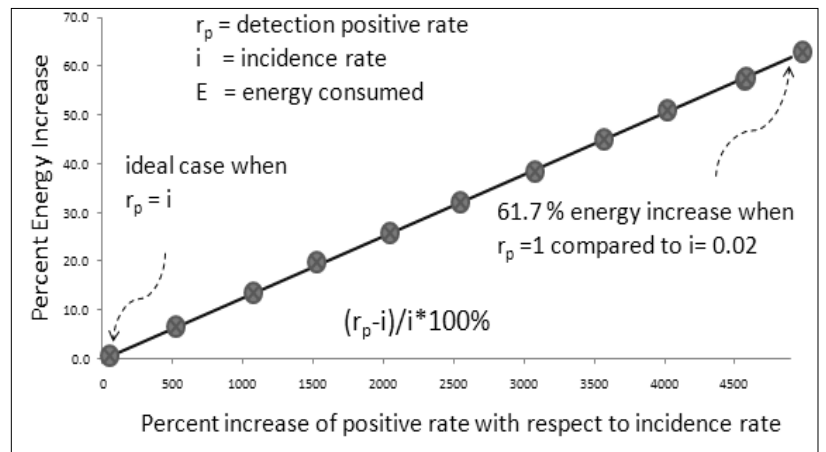

Figure 10: Energy required as positive rate varies with respect to incidence rate

\subsection{A-Fib Energy Model Versus Telemetry}

Telemetry is ubiquitous in health care monitoring. Unfortunately, it places high demand on energy consumption necessitating daily replacement of batteries in the telemetry monitoring device. If the positive rate $r_{p}$ is equal to the incidence rate $\mathrm{i}$ then if the classification detection algorithm correctly classifies $100 \%$ of the episodes of A-Fib, one may conclude that the general classification energy-aware model combined with an incidence rate delivers better results in energy consumption than the telemetry model.

$$
\begin{gathered}
\mathrm{E}_{\text {telemetry }}=\mathrm{E}_{\mathrm{ECG}_{\text {Sense }}}+\mathrm{E}_{\mathrm{ECG}_{\mathrm{Tx}}}+\mathrm{E}_{\mathrm{ECG}_{\mathrm{Rx}}}+\mathrm{E}_{\mathrm{Rep}} \\
\mathrm{E}_{\text {IncidenceRate }}=\mathrm{E}_{\mathrm{ECG}_{\text {Sense }}}+\mathrm{E}_{\mathrm{ECG}_{\mathrm{Tx}}}+\mathrm{E}_{\mathrm{ECG}_{\mathrm{Rx}}}+\mathrm{E}_{\text {classify }} \\
+\quad 0.02 * \mathrm{E}_{\mathrm{Rep}}
\end{gathered}
$$

Figure 11 depicts how the risk factor and incidence based energy efficient model combined with an A-Fib incidence rate of $2 \%$ consumes approximately $38 \%$ less energy than the telemetry model.

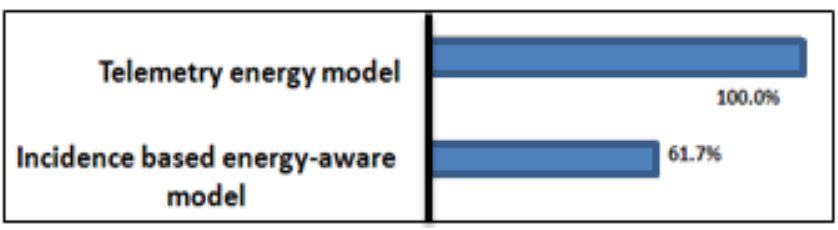

Figure 11: Comparing energy-aware model to telemetry energy model

\section{CONCLUSION}

Early recognition of A-Fib is difficult because most people are not aware of this silent rhythm disturbance [19]. A-Fib is typically diagnosed, or misdiagnosed, during a routine screening visit or during a yearly scheduled check-up, by a general practitioner or a referred cardiologist. Current cardiac A-Fib telemetry devices do not deliver continuous real-time monitoring and require a long battery life. Furthermore, because some of these solutions require patient interaction and device activation, they may become impractical when the patient is incapacitated during symptomatic periods. In this paper we design an energy efficient model for realtime monitoring and detection of cardiac A-Fib using A-Fib risk factor assessment and A-Fib incidence rate. Though our energyaware model depends on detection accuracy, the wearable computing detection model shows promising results in meeting the energy needs of real-time monitoring, detecting and reporting required in wearable computing healthcare applications.

\section{REFERENCES}

[1] http://www.OmniMedicalSearch.

[2] Domanski MJ. The epidemiology of atrial fibrillation. Coronary Artery Disease 1995;6:95-100.

[3] "Atrial Fibrillation (for Professionals)". American Heart Association, Inc. 2008-12-04.

[4] Coyne KS, Paramore C, Grandy S, Mercader M, Reynolds M, Zimetbaum P. Assessing the Direct Costs of Treating Nonvalvular Atrial Fibrillation in the United States. Value Health. Sep-Oct 2006;9(5):348-56

[5] L.Schwiebert, S. Gupta \& J. Weinmann, "Research challenges in Wireless networks of Biomedical Sensors". ACM SIGMOBILE 7/01 Rome Italy; 2001

[6] R. Bouhenguel, I. Mahgoub, and M. Ilyas, "Bluetooth Security in Wearable Computing Applications," Proceedings of the IEEE 5th International Symposium on High Capacity Optical Networks \& Enabling Technologies (HONET 2008), Penang, Malaysia, Page(s) 182 - 186, November 2008.

[7] Lasko T-A, Bhagwat J-G, Zou K-H, Ohno-Machado L. The use of receiver operating characteristic curves in biomedical informatics. J Biomed Inform 2005;38:404-15.

[8] A. Y. Benbasat and J. Paradiso. A Framework for the AutomatedGeneration of Power-Efficient Classifiers for Embedded Sensor Nodes. In SenSys '07, pages 219-232, 2007.

[9] J. Flinn and M. Satyanarayanan. Energy-Aware Adaptation for Mobile Applications. In SOSP '99, pages 48-63, October 1999

[10] Y.-T. Peng and D. Sow. Data Scaling in Remote Health Monitoring Systems. In IEEE International Symposium on Circuits and Systems (To appear), 2008.

[11] Patel, K.; Chern-Pin Chua; Fau, S.; Bleakley, C.J. ,Low power real-time seizure detection for ambulatory EEG; Complex \& Adaptive Syst. Lab., Univ. Coll. Dublin, Dublin, Ireland, Pervasive Computing Technologies for Healthcare, 2009. PervasiveHealth 2009. 3rd International Conference.

[12] Bogun F, Anh D, Kalahasty G, et al. Misdiagnosis of Atrial Fibrillation and its Clinical Consequences. Am J Med. 2004

[13] Knight BP, Michaud GF, Strickberger SA, et al. Electrocardiographic Differentiation of Atrial Flutter from Atrial Fibrillation by Physicians. J Electrocardiol 99;32:3159

[14] Poon K, Okin PM, Kligfield P. Diagnostic Performance of a Computer-Based ECG Rhythm Algorithm. J Electrocardiol 2005;38:235 8

[15] Ziad Sankari, Hojjat Adeli, HeartSaver: A mobile cardiac monitoring system for auto-detection of atrial fibrillation, myocardial infarction, and atrio-ventricular block, Computers in Biology and Medicine Volume 41, Issue 4, Pages 211-220, April 2011.

[16] Alive Heart and Activity Monitor, Alive Technologies Pty. Ltd, http://www.alivetec.com/products.htm

[17] Renate B Schnabel MD et al., Development of a risk score for atrial fibrillation (Framingham Heart Study): a communitybased cohort study, The Lancet, Volume 373, Issue 9665, Pages 739 - 745, 28 February 2009. 
[18] P. Aghera, D. Krishnaswamy, D. Fang, A. Coskun, and T. Rosing, "DynAHeal: Dynamic energy efficient task assignment for wireless healthcare systems", in Proc. DATE, 2010, pp.1661-1664

[19] Furberg CD, Psaty BM, Manolio TA, et al., Prevalence of Atrial Fibrillation in Elderly Subjects (The Cardiovascular Health Study), Am J Cardiol, 1994;74:236 41

[20] Benjamin EJ,Levy D, MD; Vaziri SM, MPH; D'Agostino $\mathrm{RB}$,Belanger AJ, Wolf PA. Independent Risk Factors for Atrial Fibrillation in a Population-Based Cohort.The Framingham Heart Study. JAMA. 1994;271:840-844

[21] Abdel Latif A, Messinger-Rapport BJ. Should Nursing Home Residents with Atrial Fibrillation be Anticoagulated? Cleve Clin J Med. Jan 2004;71(1):40-4

[22] Thaddeus R. F. Fulford-Jones, Gu-Yeon Wei, Matt Welsh, A Portable, Low-Power, Wireless Two-Lead ECG System, Div. of Engineering and Applied Sciences, Harvard University, Proceedings of the 26th Annual International Conference of the IEEE EMBS San Francisco, CA, USA Sept 1-5, 2004

[23] Mant J, Fitzmaurice DA, Hobbs FD, et al. Accuracy of Diagnosing Atrial Fibrillation On Electrocardiogram by Primary Care Practitioners and Interpretative Diagnostic Software: Analysis of Data from Screening for Atrial Fibrillation in the Elderly (SAFE) Trial. BMJ 2007;335-380.

[24] Shiyovich A, Wolak A, Yacobovich L, Grosbard A, Katz A., Accuracy of Diagnosing Atrial Flutter and Atrial Fibrillation from a Surface Electrocardiogram by Hospital Physicians: Analysis of Data from Internal Medicine Departments, Cardiology Department, Soroka University Medical Center, Ashkelon, Israel, Am J Med Sci. 2010 Oct;340(4):271-5

[25] Perez, Marco V; Dewey, Frederick E; Marcus, Rachel; Ashley, Euan A; Al-Ahmad, Amin A; Wang, Paul J, Electrocardiographic predictors of atrial fibrillation, American Heart Journal, ISSN 0002-8703, 2009, Volume 158, Issue 4, pp. $622-628$.

[26] Peter Reinelt, Georg Karth, Alexander Geppert and Gottfried Heinz, Incidence and Type of Cardiac Arrhythmias in Critically Ill Patients: a Single Center Experience in a Medical-Cardiological ICU, Intensive Care Medicine, Volume 27, Number 9, 1466-1473
[27] HRS/EHRA/ECAS Expert Consensus Statement on Catheter and Surgical Ablation of Atrial Fibrillation: Recommendations for Personnel, Policy, Procedures and Follow-Up http://www.hrsonline.org/News/Media/press releases/CSAblation.cfm

[28] V. Fuster (ed.), Hurst's The Heart, Tenth Edition New York, NY: McGraw-Hill Medical Publishing, 2001

[29] Cardionet: Charging a momitor http://www.cardionet.com/medical_06.htm

[30] R. Bouhenguel, I. Mahgoub, A Risk and Incidence Based Atrial Fibrillation Detection Scheme for Wearable Healthcare Computing Devices, 6th International Conference on Pervasive Computing Technologies for Healthcare San Diego, CA, 2012.

[31] R.Bouhenguel, I. Mahgoub, M. Ilyas, Window-Based EnergyAware Model for Real-Time Detection and Reporting of Progressive Development of Cardiac Atrial Fibrillation in Wearable Computing, Proceedings of the IEEE 8th International Symposium on High Capacity Optical Networks \& Enabling Technologies (HONET 2011), Riyadh, Saudi Arabia, 2011. IEEE, Pages: 84-88

[32] D.R. Cox, Regression Models and Life-Tables, journal articles in statistics and medicine published in 1972.

[33] UCI Machine Learning Repository, 2007

[34] PhysioBank, ANSI/AAMI EC13 Test Waveforms, http://physionet.org/physiobank/database/aami-ec13/

[35] Fuster V, Rydén LE, Cannom DS, et al., ACC/AHA/ESC 2006 Guidelines for the Management of Patients with Atrial Fibrillation. Circulation , 2006

[36] Wolf PA, Kannel WB, McGee DL, et al. Duration of Atrial Fibrillation and Imminence of Stroke: The Framingham Study. Stroke. 1983;14(5):664-667

[37] Andrew D. Krahn, MD, Jut-e Manfreda, MD, Robert B. Tate, MSc, Francis A.L. Mathewson, MD, T. Edward Cuddy, The Natural History of Atria1 Fibrillation: Incidence, Risk Factors, and Prognosis in the Manitoba Follow-Up Study, MD, Winnipeg, Canada, The American Journal of Medicine, May 2005 